\title{
Predicted Multi-variable Intelligent Matching Pursuit Algorithm for Image Sequences Reconstruction Based on $l_{0}$ Minimization
}

\author{
Dan Li, Qiang Wang Member IEEE, Yi Shen Member IEEE ${ }^{1}$ \\ Control Science and Engineering, Harbin Institute of Technology, \\ No.92, West Da-Zhi Street, Nangang District, Harbin, China, 150001 \\ lidanhit@163.com,wangqiang@hit.edu.cn, shen@hit.edu.cn
}

\begin{abstract}
In this paper, we study the problem of reconstructing image sequences which satisfy the conditions that (a) the sparsity level is high in the wavelet domain and (b) the sparsity pattern of adjacent images changes very slowly. The idea of the proposed method predicted multi-variable intelligent matching pursuit (PMIMP) algorithm is to use the estimated support collection of the previous image as prior information and then utilize the prior information to guide the current image reconstruction by solving $l_{0}$ minimization. Multi-variable scheme is used to sample image sequences to enhance the guidance of prior information and improve the reconstruction accuracy with fewer measurements. $l_{0}$ minimization is an NP-hard problem that requires exhaustively listing all possibilities of the original signal and is difficult to be achieved by traditional algorithms. To solve it, we take advantage of the intelligent optimization algorithm which is famous for its global searching ability and superior performance in solving combinatorial optimization problems. To improve the reconstruction speed, matching strategies of greedy algorithm, which performs quite well in reconstruction speed, are utilized to design the updating mechanism of PMIMP. As the sparsity level is hard to be estimated in image sequences reconstruction, we propose a novel optimization function which does not need the sparsity level known as a prior. We illustrate the reconstruction performance of our proposed method PMIMP on several image sequences and compare it with the
\end{abstract}


state-of-the-art algorithms. The experimental results demonstrate that PMIMP achieves the best reconstruction performance in both PSNR, SSIM and visual quality with fewer measurements.

Keywords: Image sequences reconstruction, Prior information, Multi-variable sampling, $l_{0}$ minimization, Intelligent optimization algorithm, Matching strategies.

\section{Introduction}

Image sequences, such as computed tomography $(\mathrm{CT})$ image sequences and magnetic resonance (MR) image sequences, satisfy the conditions that (a)the sparsity level of each image is high in the wavelet domain and (b) the sparsity pattern of adjacent images changes very slowly. So compressive sensing (CS) $[1,2,3]$ can be used to reconstruct the original sequences from a much smaller number of measurements. One kind of the existing methods $[4,5,6]$ for image sequences reconstruction treats all the frames in the sequences as a single spatiotemporal signal, which leads to a very high computational complexity. An alternative kind of methods would apply compressive sensing reconstruction to each frame separately, which has a low computational complexity but needs more measurements to achieve the accurate reconstruction.

Image sequences reconstruction based on $l_{0}$ minimization is an NP-hard problem which is difficult to be achieved by traditional algorithms. Methods available for solving the $l_{0}$ minimization directly are greedy algorithms [7], such as orthogonal matching pursuit (OMP) algorithm [8], subspace pursuit (SP) algorithm [9], stagewise orthogonal matching pursuit (StOMP) algorithm[10], polytope faces pursuit (PFP) algorithm [11], backtracking-based matching pursuit (BAOMP) algorithm [12], sparsity adaptive matching pursuit (SAMP) algorithm [13] and compressive sampling matching pursuit (CoSaMP) algorithm [14]. Suppose the sparsity level is known as a prior, the purpose of greedy algorithm is to estimate the support collection and its corresponding coefficients using the least square method based on the residual correlation. However, the 
residual correlation just can reflect the correlative degree between the coefficients and the measurement signal, but cannot indicate that the larger the residual correlation is, the larger the coefficient should be. Another common shortcoming of greedy algorithm is that it is more likely to fall into a suboptimal solution. While intelligent optimization algorithm $[15,16,17]$ is famous for its global searching ability and superior performance in solving combinatorial optimization problems, it is deserved to be used to solve $l_{0}$ minimization essentially. Simulated annealing algorithm is widely used for CS reconstruction by $l_{0}$ minimization, such as hybrid simulated annealing thresholding (HSAT) algorithm [18], simulated annealing algorithm for sparse reconstruction (SASR) [19] and heuristic search algorithm for multiple measurement vectors problem (HSAMMV) [20]. The proposed methods in [18, 19, 20] are both single-cycle, which are time-consuming and easy to find a sub-optimal solution when the measurement number is relatively small. Also, the precondition of $[18,19,20]$ is that the sparsity level must be known as a prior. However, the sparsity level is hard to be estimated in many reconstruction problems. In our previous work $[21,22]$, we propose two reconstruction methods based on the combination of genetic algorithm and bacterial foraging optimization (GA-BFO) algorithm and artificial immune algorithm (AIA), which perform quite well in reconstruction accuracy. Nevertheless, the computational complexity of the two methods are high, which leads to the slow reconstruction speed.

Prior information, which plays an important role in image processing, can not only improve the efficiency of the reconstruction algorithms but also reduce the computational complexity to accelerate the reconstruction speed. Many methods using image structures as the prior information were proposed. [23] proposed a novel framework for image super-resolution using an over-complete dictionary based on effective image-representation, such as edges, contours and high-order structures. A new framework for single image and video superresolution is proposed in [24], which aims to employ the geometric details of the image as the prior information to minimize the possible artifacts. [25] also used some image structures to calculate the image priority, such as the novel 
features derived from an integral-image and the integral-image-based temporal gradients, which can extracts semantically important frames from the video to reduce the bandwidth requirement. If the sparsity pattern of adjacent images in the image sequences changes very slowly, it is natural to apply some information of the previous image as the prior information to guide the current image reconstruction. In [26], the authors proposed a method called Kalman filtered compressive sensing (KF-CS), which runs a reduced order Kalman filter with the current estimated support and estimates new additions to the support set by applying CS to the Kalman innovations or filtering error. [27] proposed a least square method (LS-CS) for image sequences reconstruction by using compressive sensing on the least square residual computed by the estimated support collection of the previous image instead of using compressive sensing on the measurement data. The modified compressive sensing method (modified-CS) was proposed for image sequences reconstruction with partially known support in [28]. The purpose of the modified-CS is to solve a convex relaxation of the problem that find the signal which satisfies the data constraint and is the sparsest outside the known support. Afterwards, some more powerful methods such as modified-CS-residual [29, 30] and regularized modified-CS [31, 32] were developed. Other recent work which applies compressive sensing to measure the difference signal and then reconstructs the difference signal was proposed in [33]. Also, the weighted-CS proposed in[34] used the estimated previous frame to extract an estimated probability model and then used the model to guide the reconstruction process.

In this paper, we propose a novel method called predicted multi-variable intelligent matching pursuit (PMIMP) for image sequences reconstruction. PMIMP, which aims to estimate the support collection of the current original image intelligently and accurately based on the limited number of measurements and the prior information, addresses the $l_{0}$ minimization problem essentially. First of all, based on the fact that the sparsity pattern of adjacent images is correlative and changes slowly, we set the estimated support collection of the previous image as the prior information. After that, we use the prior informa- 
tion to guide the current image reconstruction, which can not only reduce the computational complexity but also reconstruct the current image accurately using fewer measurements. Also, multi-variable scheme, which takes advantage of the statistic dependency among the wavelet coefficients, is used to sample the original image to enhance the guidance of the prior information and improve the reconstruction performance significantly. Then, as the sparsity level is hard to be estimated in image sequences reconstruction, we propose a novel optimization function which does not need the sparsity level known as a prior. In addition, we utilize the advantage of intelligent optimization algorithm in solving combinatorial optimization problems to solve the $l_{0}$ minimization essentially, which is also beneficial to finding the global optimal solution (support collection). To improve the reconstruction speed, matching strategies of greedy algorith$\mathrm{m}$, which performs quite well in reconstruction speed, are introduced to design the updating mechanism of PMIMP. As PMIMP can solve $l_{0}$ minimization essentially and find the global optimal solution, it improves the reconstruction accuracy significantly for image sequences. By means of setting the estimated support collection as the prior information and introducing matching strategies to design the updating mechanism, PMIMP also has a reasonable reconstruction speed, especially when the sparsity pattern changes slowly. Experiments on phantom image sequence, brain CT image sequence and brain MR image sequence based on PMIMP and other state-of-the-art algorithms, including OMP, CoSaMP, edge based matching pursuit algorithm (EMPA) [35], Kalman Filtered compressive sensing (KF-CS) method, least square residual compressive sensing (LS-CS) method, KFLS-CS and Modified-CS, are used to illustrate the superiorities of the proposed method PMIMP.

In accordance with the process of CS, the major contributions of this paper are fourfold:

1. We use the estimated support collection of the precious image as the prior information to guide the current image reconstruction, which can not only reduce the computational complexity but also reconstruct the current 
image accurately using fewer measurements.

2. We use multi-variable scheme to sample each frame of the original image sequences, which is beneficial to enhancing the guidance of prior information and improving the reconstruction performance significantly.

3. As the sparsity level of image sequences is hard to be estimated, we develop a novel optimization function for image sequences reconstruction when the sparsity level is unknown as a prior.

4. We solve the $l_{0}$ minimization problem essentially by taking advantage of intelligent optimization algorithm in combinatorial optimization problems and improve the reconstruction speed by introducing matching strategies of greedy algorithm.

The reminder of this paper is organized as follows: In section 2, the related works are provided for an easy understanding of our proposed method. In section 3, the framework of PMIMP and the computational complexity analysis are provided. Experimental results are given in Section 4 to illustrate the performance of the proposed method PMIMP. Section 5 concludes this paper.

\section{Related works}

\subsection{Image sequences reconstruction}

Suppose the original image sequence is represented by $X:=\left[x_{1}, x_{2}, \ldots, x_{n}\right]$, where $x_{i}, i=1,2, \ldots, n$ represents the $i t h$ frame of the original image sequence. In compressive sensing framework, each frame of image sequence $x_{i}, i=1,2, \ldots n$ can be represented sparsely in the sparse basis $\psi$ and is sampled using a measurement matrix $\phi$ with size of $m * N(m<N)$ which is uncorrelated to $\psi$ and satisfies restrict isometry property (RIP). Then we can obtain the measurement signal $y_{i}, i=1,2, \ldots, n$ using Equ.(1).

$$
y_{i}=\phi * x_{i}=\phi * \psi * s_{i}
$$


where, $s_{i}$ is the sparse representation of $x_{i}$ in the sparse basis $\psi$.

To simplify Equ.(1), $x_{i}$ is assumed to be sparse in the following sections. The sparse reconstruction problem [36, 37] can be implemented by optimizing a constrained optimization problem of sparsity, i.e. finding the sparsest signal $x_{i}$ among all signals satisfying $y_{i}=\phi * x_{i}$, which can be described in Equ.(2).

$$
\operatorname{recx}_{i}=\operatorname{argmin}\left\|x_{i}\right\|_{0} \quad \text { subject to } \quad y_{i}=\phi * x_{i},
$$

where, $\left\|x_{i}\right\|_{0}$ represents the $l_{0}$ norm of $x_{i}$, which is defined by the number of nonzero elements of $x_{i}$. Euq.(2) can be also written as the form of Equ.(3).

$$
\operatorname{rec} x_{i}=\operatorname{argmin}\left\|x_{i}\right\|_{0}+\lambda *\left\|y_{i}-\phi * x_{i}\right\|_{2}^{2},
$$

where $\lambda$ is a constant between 0 and 1, which is used to balance the trade-off between the sparsity and reconstruction error.

If the sparsity level is known as a prior, the image sequences reconstruction problem described in Equ.(3) can be handled using a two-step method which has been used in many greedy algorithms. The first step is to obtain the estimated support collection $I$ of the original signal, where length $(I)=K$ and $K$ represents the sparsity level. Then the estimated solution can be obtained using the least square method in the second step, which is shown in Equ.(4).

$$
\operatorname{rec}_{I}=\Phi_{I}^{\dagger} * y \quad \text { and } \quad r e c_{S-I}=0 .
$$

where $S=[1,2, \ldots, N]$ indicates the index of each entry of the original signal and $N$ represents the length of the original signal. Suppose $K$ entries are selected from $S$ to generate the support collection $I, x_{I}$ consists the entries of $x$ indexed by $i \subset I$ and the matrix $\phi_{I}$ is composed of the columns of $\phi$ with indexes $i \subset I$. $\phi_{I}^{\dagger}$ represents the pseudo-inverse operation of the measurement matrix which is calculated by $\phi^{\dagger}=\left(\phi^{\top} * \phi\right)^{-1} * \phi^{\top}$, where $\top$ represents matrix transportation.

As described in [19], the following Lemma 1 is used to obtain the optimization function of the two-step method. 
Lemma 1: suppose that $x^{*}$ with support $I^{*}$ is the unique solution of Equ.(3), and the sparsity level $K$ is known as a prior and satisfies $K<\operatorname{spark}(\phi) / 2$, where $\operatorname{spark}(\phi)$ is the smallest number of columns from $\phi$ that are linearly dependent. Then we have $x=x^{*}$ if and only if $\phi_{I} * \phi_{I}^{\dagger} * y=y$, where $I$ satisfying length $(I)=K$ is the estimated support collection and the estimated solution $x$ is obtained using Equ.(4).

From Lemma 1, if the sparsity level is known as a prior and satisfies $K<$ $\operatorname{spark}(\phi) / 2$, we can obtain the solution by searching the support collection $I$ and then calculating $\phi_{I} * \phi_{I}^{\dagger} * y=y$. So the optimization function can be defined as Equ.(5).

$$
f(I)=\left\|\Phi_{I} * \Phi_{I}^{\dagger} * y-y\right\|_{2}
$$

As described above, the estimated support collection can be obtained by optimizing Equ.(6) and then the estimated solution can be calculated by Equ.(4).

$$
\min _{I} f(I)
$$

\subsection{Multi-variable scheme}

Parallel framework is beneficial to reducing the computational complexity and speeding up the method. For example, $[38,39,40]$ respectively proposed a parallel framework for HEVC to decouple ME for different partitions on manycore processors, which can speed up the HEVC method significantly. Multivariable scheme $[35,41]$, which was developed to take advantage of the statistic dependency among the wavelet coefficients in CS reconstruction, is a parallel framework and is an extension of unit-variable sampling. Utilizing the statistical dependency of the wavelet coefficients in neighbourhood, we estimate a small neighbourhood of wavelet coefficients instead of estimating one wavelet coefficient. An example to describe the process of the two sampling scheme is shown in Fig. 1.

The unit-variable sampling scheme in traditional CS first rearranges the wavelet coefficients to a vector in which each coefficient is independent to others, 

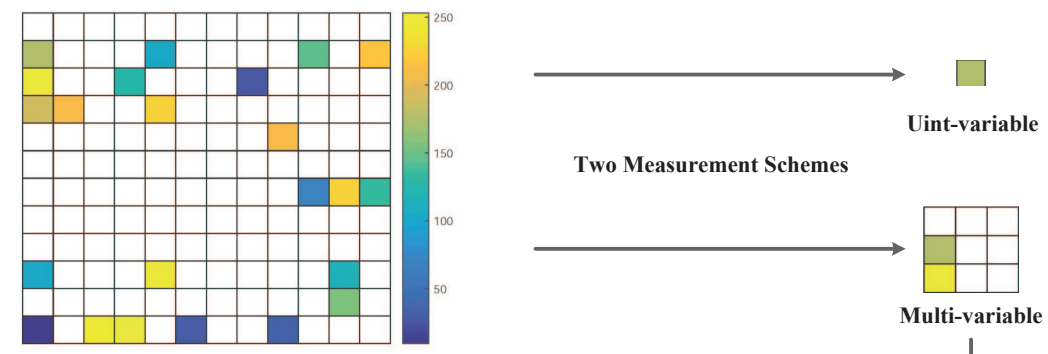

Two Measurement Schemes

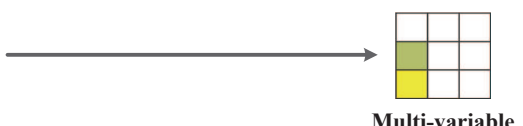

Original Signal
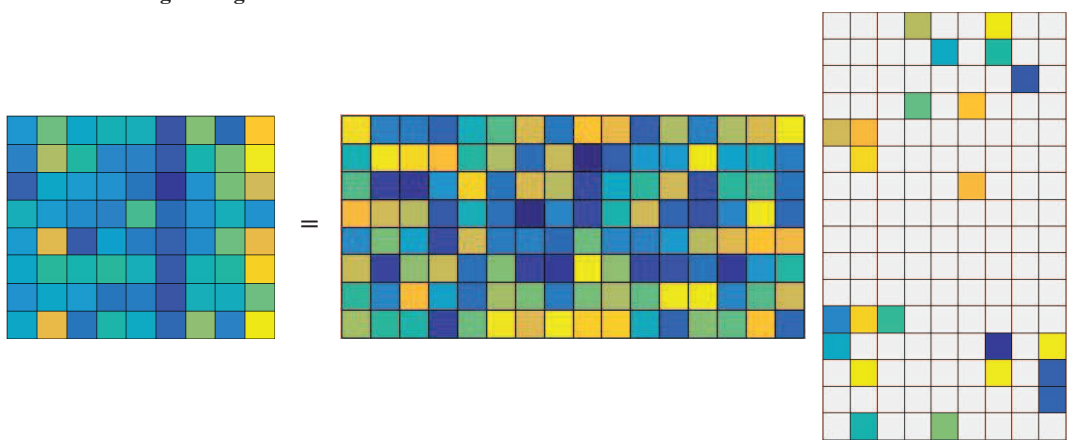

Measurement Signal

Measurement Matrix

Multi-variable Signal

Figure 1: An example to describe the multi-variable sampling scheme. The size of the original signal is $12 * 12$. unit-variable sampling scheme is to reshape the original signal as a vector with size of $144 * 1$ and sample the vector. In multivariable scheme, suppose the size of neighbour is set as $3 * 3$, the original signal can be reshaped as a multi-variable signal with size of $16 * 9$. Then the multi-variable signal can be sampled using a Gaussian random matrix.

and then samples it using a measurement matrix. In the multi-variable scheme, we first partition the wavelet coefficients into small neighbourhoods with size of $d * d$, then $X$ is reshaped to a multi-variable form $X^{\prime}$ with size of $N^{\prime} * d^{2}$, where $N^{\prime}=N / d$ and the $i$ th row of $X^{\prime}$ represents the ith neighbourhood. In the end, we obtain the multi-variable measurement signal $Y$ by sampling $X^{\prime}$ using a measurement matrix $\Phi$, namely $Y=\Phi * X^{\prime}$. After multi-variable sampling, $X^{\prime}$ can be jointly reconstructed from the measurement image $Y$ and the measurement matrix $\Phi$. The formulation of multi-variable joint reconstruction can be represented by Equ.(7). 


$$
\min _{X^{\prime}} \operatorname{supp}\left(X^{\prime}\right) \quad \text { subject to } \quad Y=\Phi * X^{\prime} \text {, }
$$

where $\operatorname{supp}\left(X^{\prime}\right)=\sum_{j}^{N} \operatorname{supp}\left(X^{\prime}(:, j)\right), X^{\prime}(:, j)$ represents the $j$ th column of $X^{\prime}$ and $M$ represents the number of columns.

\section{The proposed PMIMP}

In this section, we first introduce the prior information and the novel optimization function, and then develop the framework of the PMIMP for image sequences reconstruction. In the end, we analysis the computational complexity.

\subsection{Prior information}

Considering the slow change of the sparsity pattern of adjacent images, the estimated support collection of the previous image can be set as the prior information to guide the current image reconstruction. The estimated support collection of the previous image is defined as nonzero set and indexes out of the estimated support collection consist of the zero set for the current image reconstruction. In the process of the current image reconstruction, the nonzero set replaces the set $S$, which can reduce the size of solution set to reduce the computational complexity. Under the guidance of prior information, our method PMIMP is more easier to find the global optimal solution with fewer measurements.

To enhance the accuracy of the guidance of the prior information, the multivariable scheme is used to sample the original image. The multi-variable sampling scheme and joint reconstruction can significantly enhance the similarity of the adjacent images in image sequences, which is beneficial to predicting and guiding the current image reconstruction. An example of nonzero indexes of two adjacent brain CT images is shown in Fig.2. The size of the sampling area is $8 * 8$ and that of the neighbourhood is $2 * 2$. So there are 64 components in unit-variable vector and 16 components in multi-variable vector. From Fig.2, we can see that the percentage of the same indexes between the two adjacent 


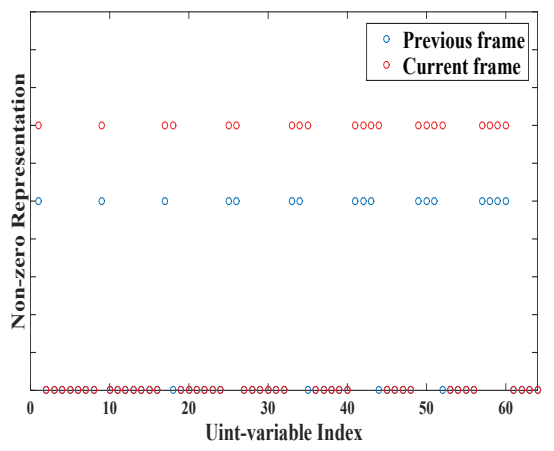

(a)

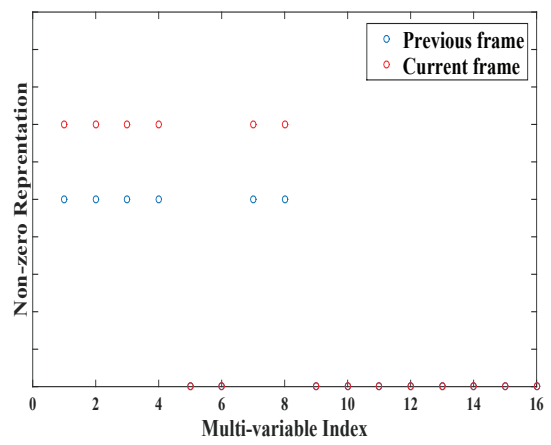

(b)

Figure 2: The similarity of two adjacent images. (a) Unit-variable sampling, (b) Multi-variable sampling. The abscissa represents the indexes and the ordinate represents the values of the indexes (zero or non-zero). The red circles represent the values of the precious image and the blue ones represent the values of the current image.

images using the unit-variable sampling scheme is $93.75 \%$, while that using the multi-variable sampling scheme can reach $100 \%$. The superior performance of multi-variable sampling scheme can enhance the guidance of prior information and improve the reconstruction performance.

\subsection{The novel optimization function}

As described in section 2.1, if the sparsity level is known as a prior, the optimization function can be set as Equ.(5). However, the sparsity level is always hard to be estimated accurately in many reconstruction problems. To solve this problem, we propose a novel optimization function which does not need the sparsity level known as a prior. Before giving Theorem 1, we list Lemma 2, which can be used to prove Theorem 1.

Lemma 2 Let the columns of $\phi$ be in general position. Let $I_{S}$ denote the support of $x_{S}$. Suppose that the support collection $I_{0}$ of $x_{0}$ is a subset of $I_{S}$. Then we have the perfect recovery:

$$
x_{S}=x_{0}
$$


From Lemma 1, we can see that the support collection $I$ satisfies $\| \Phi_{I} * \Phi_{I}^{\dagger} *$ $y-y \|_{2}=0$. Define the collection $I_{m m}$ (where $K \leq m m \leq m$ ), which denotes the mm-cardinality subsets of the set $S$. It can be inferred from Lemma 2 that if the support collection $I$ is a subset of the collection $I_{m m}, I_{m m}$ satisfies the condition that $\left\|\Phi_{I_{m m}} * \Phi_{I_{m m}}^{\dagger} * y-y\right\|_{2}=0$. Also, $f\left(I_{m m}\right)=\left\|\Phi_{I_{m m}} * \Phi_{I_{m m}}^{\dagger} * y-y\right\|_{2}$ satisfies that $f\left(I_{m m}\right) \geq 0$, so we propose a novel optimization function in Theorem 1 .

Theorem 1: Suppose the sparsity level is unknown in advance, the collection $I_{m m}$, which contains the support collection of the original signal, can be estimated by optimizing the optimization function described in Equ.(9) and the reconstructed signal can be obtained by Equ.(10).

$$
\begin{gathered}
\min _{I_{m m}} f\left(I_{m m}\right)=\min _{I_{m m}}\left\|\Phi_{I_{m m}} * \Phi_{I_{m m}}^{\dagger} * y-y\right\|_{2}, \\
\operatorname{recx}_{I_{m m}}=\Phi_{I_{m m}}^{\dagger} * y \quad \text { and } \quad r e c_{S-I_{m m}}=0 .
\end{gathered}
$$

Finally, the fitness function of the proposed method PMIMP can be set as $f\left(I_{m m}\right)=\left\|\phi_{I_{m m}} * \phi_{I_{m m}}^{\dagger} * y-y\right\|_{2}^{2}$. Each individual represents a possible solution $I_{m m}$ and the solution set of the optimization function is their searching area.

\subsection{The framework of PMIMP}

After multi-variable sampling, each column of $X^{\prime}$, which is represented as a signal $x$, can be reconstructed through the corresponding column of $Y$ and the measurement matrix $\Phi$. We use the intelligent optimization algorithm, combining genetic algorithm [42] with simulated annealing algorithm [43], to estimate the support collection and its corresponding coefficients intelligently based on the guidance of prior information. Genetic algorithm (GA) is a random searching algorithm achieved by simulating natural selection and genetic mechanisms, which performs better in global searching. The main operations of GA are selection, crossover and mutation. However, GA has an obvious shortcoming, i.e. it is time-consuming in practical application. To overcome the shortcoming of GA, simulated annealing ( $\mathrm{SA}$ ) algorithm is introduced. SA is an essentially iterative 
random searching procedure and implements Metropolis rule in each iteration to update the group. Based on the Metropolis rule, SA permits accepting a less optimal solution and also some random solutions with positive probability. Combining GA with SA can make the algorithm have both powerful global and local searching ability, which is more likely to find the global optimal solution. Nevertheless, the computational complexity of intelligent optimization algorith$\mathrm{m}$ is always high, which leads to a slow convergence speed. Considering the reconstruction speed, we design the updating mechanism utilizing matching strategies of greedy algorithm which performs quite well in reconstruction speed. We initial the individuals using the matching strategies of SP and OMP. The updating mechanism of iterations is based on the matching strategies of OMP and CoSaMP.

The procedure of PMIMP is shown in Algorithm 1. There are five major elements, including prior information, fitness function, initialization, iteration and terminal condition, which are described in details below.

(1)Prior information. As the sparsity level of adjacent images changes very slowly, we set the estimated support collection of the previous image as the prior information to guide the current image reconstruction. This means that the nonzero set $S$ obtained from the previous image is set as the searching area of the current image.

(2)Fitness function. As proposed in section 3.2, the optimization function is set as $f\left(I_{m m}\right)=\left\|\phi_{I_{m m}} * \phi_{I_{m m}}^{\dagger} * y-y\right\|_{2}^{2}$.

(3)Initialization. We initialize the individuals based on matching strategies of OMP and SP. The rule of the initialization is: 1) calculate $x_{1}=\phi^{\dagger} * y$ and select the $m m$ indexes with bigger numerical value as the first individual, 2) assign the column corresponding to the index with the biggest numerical value zero, 3) calculate $x_{2}=\phi^{\dagger} * y$ and select the $m m$ indexes with bigger numerical value as the second individual, 4) the afterwards individuals are all initialized based on step 2) to step 3).

(4)Iteration. The proposed PMIMP is a two-cycle intelligent searching algorithm, where the outer loop is GA which has strong global searching ability 


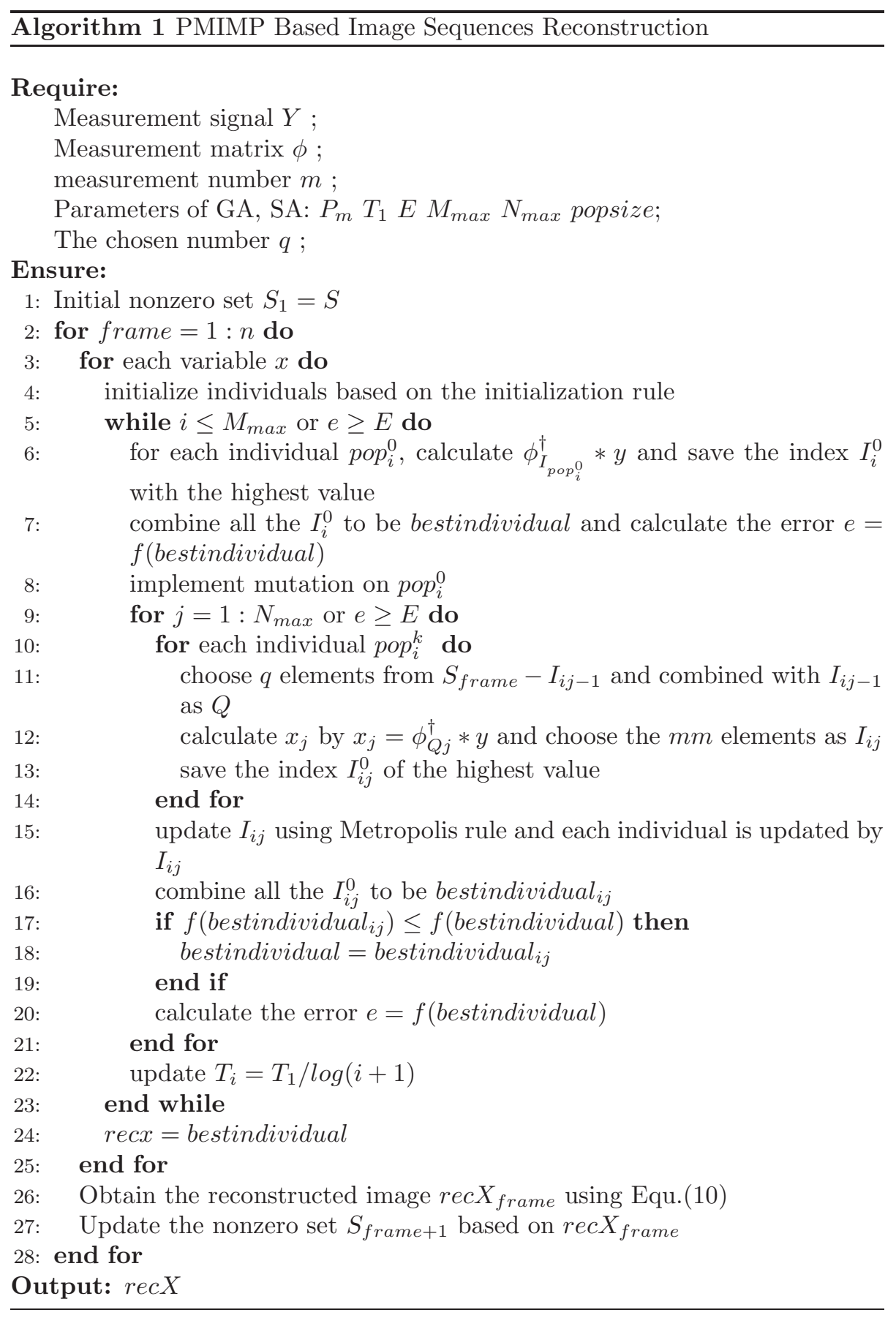


and the inner loop implemented is SA which performs better in local searching.

(a) Strategies in the outer loop. 1) Calculate the fitness of each individual based on the fitness function $f\left(I_{m m}\right)=\left\|\phi * \phi_{I_{m m}}^{\dagger} * y-y\right\|_{2}^{2}$ and save the indexes $I_{i}^{0}$ of the highest value for each individual, 2) Combine all the $I_{i}^{0}$ to be the bestindividual, 3) Mutation operation is implemented to all the individuals. Firstly, calculate $x_{I_{m m}}$ for each individual based on $x_{I_{m m}}=\phi_{I_{m m}}^{\dagger} * y$. Then remain the indexes whose numerical value are bigger and select randomly from $S-I$ to take place the smaller ones.

(b)Strategies in the inner loop. 1) select q elements from $S-I_{i j-1}$ and combine them with $I_{i j-1}$ to conduct $\left.Q, 2\right)$ calculate $x_{Q}=\phi_{Q}^{\dagger} * y$ and choose the $m m$ bigger elements from $x_{Q}$ as the new generated individual, 3) save the indexes $I_{i j}^{0}$ with the highest value, 4) calculate the fitness of both the new individual and the old individual, and then select one individual based on Metropolis rule, 5) combine all the indexes $I_{i j}^{0}$ to be bestindividual ${ }_{i j}$ and com-

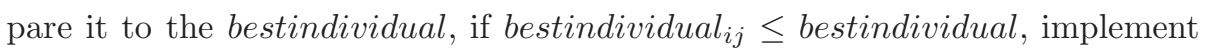
bestindividual $=$ bestindividual $_{i j}$.

Metropolis rule is that: if $p \leq \eta$, where $p$ is calculated by Equ.(11) and $\eta$ is a random number chosen in $[0,1]$, select the new individual, otherwise, select the old individual. The annealing temperature which is calculated by $T_{i}=T_{1} / \log (i+1)$ is updated in the outer loop.

$$
p=\min \left(1, \exp \left(-\left(f\left(I_{i j}\right)-f\left(I_{i j-1}\right)\right) / T_{i}\right)\right) .
$$

(5)Terminal condition. The terminal condition of PMIMP is that the number of iterations reaches the maximum number or the error is smaller than the minimum value $E$.

If the terminal condition is satisfied, the obtained bestindividual is the estimated support collection of the current signal, and then the reconstructed signal can be calculated by Equ.(10). Once the estimated support collection of the current image is obtained, it can be set as the prior information to guide the next image reconstruction. 


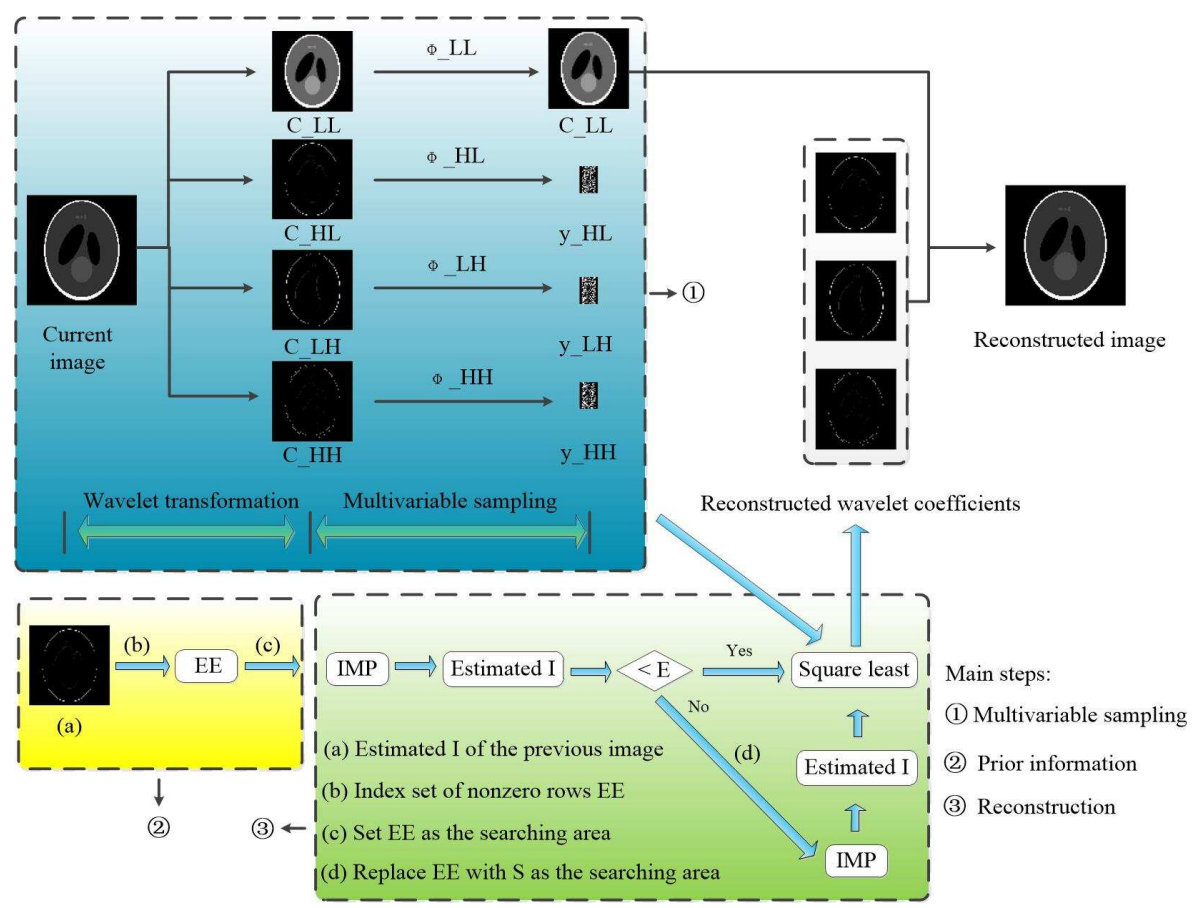

Figure 3: The overall flowchart of PMIMP for image sequences reconstruction.

The overall flowchart is shown in Fig.3, where the reconstructed image will be the prior information of the next image reconstruction. The main steps of Fig.3 are corresponding to the multi-variable sampling scheme, the prior information extracted from the previous image and the process of estimating the support collection of the current image.

\subsection{Computational complexity analysis}

From Algorithm 1, we find that the main computational complexity lies in the initialization and the iteration as they both have matrix multiplication. The computational complexity of matrix multiplication is upper bounded by $O\left(N^{3}\right)$. In the initialization, matrix multiplication is used to generate $\mathrm{mm}$ initial individuals, so the computational complexity of the initialization can be calculated by $\sum_{i=0}^{m m-1} O(N-i)^{3}$. In the iteration, matrix multiplication lies in step 7 of the outer loop and step 14 of the inner loop. The number of 
iterations is bounded by $M_{\max } * N_{\max }$ and a total of $m m$ individuals compute simultaneously. So the computational complexity of PMIMP can be calculated by Equ.(12).

$$
\sum_{i=0}^{m m-1} O(N-i)^{3}+m m * M_{\max }\left(N_{\max }+1\right) * O\left(N^{3}\right)
$$

As shown in Equ.(12), the computational complexity is influenced by the number of iterations significantly. So if we want to reduce the computational complexity of PMIMP, some methods which can reduce the number of iterations should be introduced.

\section{Experimental results}

\subsection{Experimental Configuration}

In this section, we conduct CS reconstruction experiments on three image sequences to verify the superiority of the proposed method PMIMP. The test image sequences are phantom image sequence, brain $\mathrm{CT}$ image sequence and brain MR image sequence, which are shown in Fig.4, Fig.5 and Fig.6. Phantom image sequence is a three-dimensional analogue of MATLAB Shepp-Logan phantom. The brain CT image sequence is downloaded at http://lgdv.cs.fau.de/External/vollib/ and the brain MR image sequence is downloaded at http ://decsai.ugr.es/cvg/dbimagenes/. The measurement matrix $\phi$ is obtained by extracting $m$ rows of the $N * N$ orthogonal random matrix. Suppose the measurement is noiseless in all the experiments.

We use the neighbourhood with size of $4 * 4$ in the multi-variable scheme. In order to improve the reconstruction speed, a block sampling scheme [44] is introduced in the following experiments, where the original images are blocked by windows with the size of $32 * 32$. We obtain the measurements from the subblocks and reconstruct these subblocks respectively. We conduct all the reconstruction experiments based on the proposed method PMIMP with the fixed parameters $M_{\max }=20, N_{\max }=100, E=10^{-5}$. All the experiments are 
implemented in Matlab 2011a on a PC with 3.2GHZ Intel Core $i 5$ processor and 8.0GB memory running the Windows 7 system. Here, the compared algorithm OMP is implemented using SolveOMP in SparseLab toolbox [45].

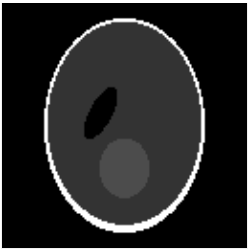

(a)

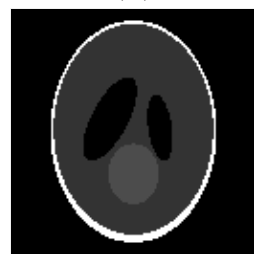

(e)

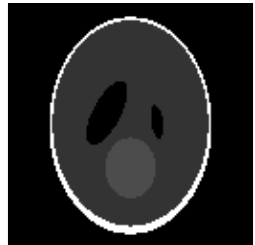

(b)

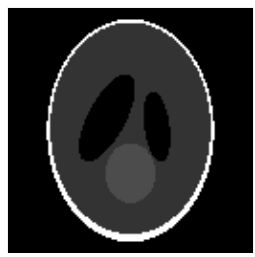

(f)

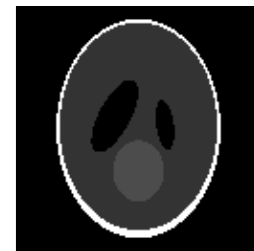

(c)

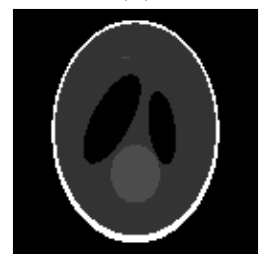

(g)

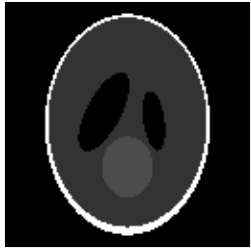

(d)

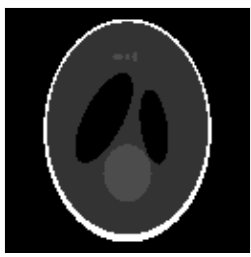

(h)

Figure 4: The test phantom image sequence with size of $128 * 128 * 8$.

\subsection{Experimental results and analysis}

In this experiment, the size of phantom image sequence and brain $\mathrm{CT}$ image sequence are $128 * 128 * 8$ and that of the brain MR image sequence is $256 * 256 * 8$. The test image sequences are all famous for their high sparsity in wavelet domain and the sparsity pattern of adjacent images in the test image sequences changes very slowly. The proposed method PMIMP is compared with seven state-of-the-art reconstruction algorithms, including OMP [8], CoSaMP [14], EMPA[35], Kalman Filtered CS (KF-CS)[26], Least Square CS (LS-CS) [27], KFLS-CS and Modified-CS [28]. Four evaluation indexes, including PSNR, Structural Similarity (SSIM) index measurement [46], Runtime and visual quality, are adopted to illustrate the superior performance of the proposed PMIMP. The PSNR is calculated by Equ.(13) and SSIM is calculated based on [46], where $\max (x)$ is the largest numerical pixel of the original image, $[a, b]=\operatorname{size}(x)$ and error is the relative error between the reconstructed image 


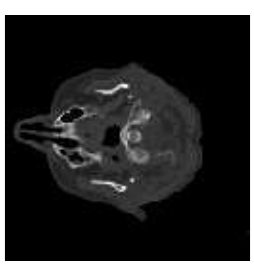

(a)

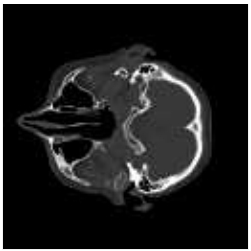

(e)

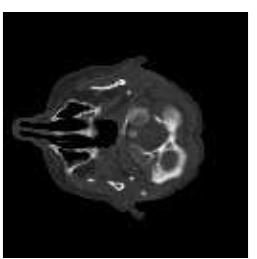

(b)

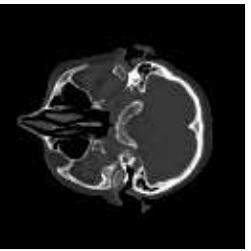

(f)

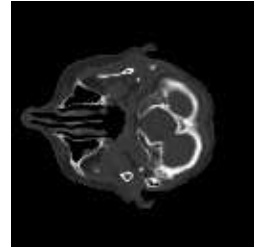

(c)

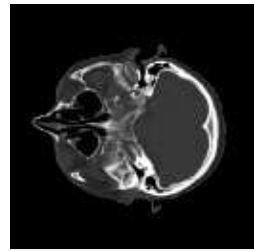

$(\mathrm{g})$

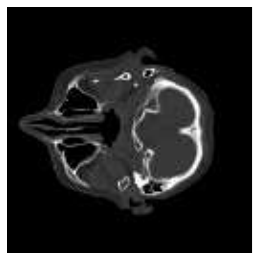

(d)

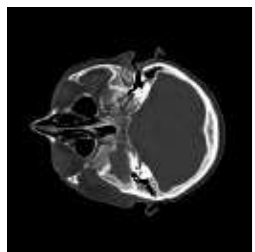

(h)

Figure 5: The test brain CT image sequence with size of $128 * 128 * 8$.

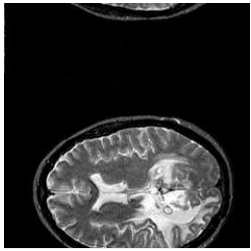

(a)

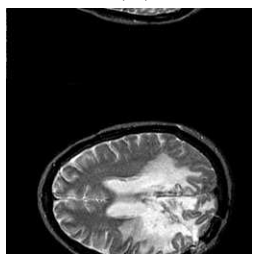

(e)

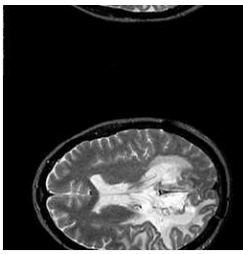

(b)

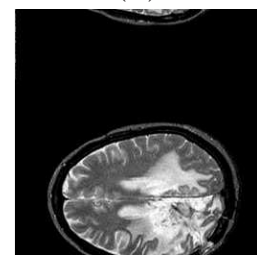

(f)

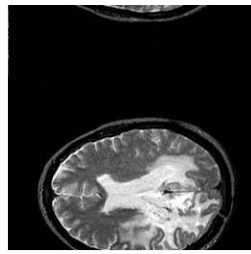

(c)

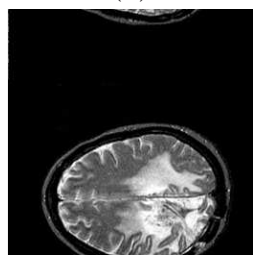

(g)

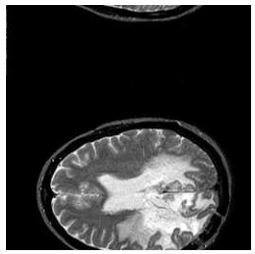

(d)

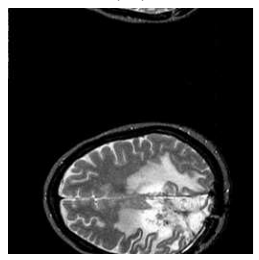

(h)

Figure 6: The test brain MR image sequence with size of $256 * 256 * 8$. 
and the original image. A higher PSNR indicates smaller errors and a SSIM value much closer to 1 indicates that the reconstructed image is more similar to the original image. The phantom image sequence reconstruction are processed with the measurement rate fixed as 0.3 and that of brain $\mathrm{CT}$ image sequence and brain MR image sequence is fixed as 0.4. Experimental results of reconstruction performance comparison between PMIMP and the aforementioned algorithms in terms of PSNR, SSIM and Runtime are shown in Table 1, Table 2, Table 3. The visual comparison for the $6 t h$ image is shown in Fig.7.

$$
P S N R=10 * \log _{10}\left(\max (x)^{2} /(\operatorname{error} / a / b)\right)
$$

From Table 1, we can find that our proposed method PMIMP achieves the highest PSNR and the highest SSIM, namely the best reconstruction accuracy, for all the frames of phantom image sequence. Table 2 and Table 3 also show that PMIMP achieves the best reconstruction performance for all the frames of brain CT image sequence and brain MR image sequence. Obviously, PMIMP improves the reconstruction accuracy significantly for the previous few images in all the test image sequences. In the case of the later few images, PMIMP takes on the appearance that PSNR is several $d B$ higher than that of those compared seven algorithms. The main reason is that PMIMP can solve $l_{0}$ minimization essentially by estimating the support collection of the original signal intelligently and accurately. Also, based on the superior performance of intelligent optimization algorithm in global searching, PMIMP is more likely to find the global optimal solution. However, those seven compared algorithms are more possible to fall into sub-optimal solutions. In addition, under the guidance of the estimated support collection of the previous image, PMIMP can reconstruct images accurately with fewer measurement. Moreover, the multi-variable sampling scheme also can improve the reconstruction performance significantly when the measurement rate is relatively small.

Considering the reconstruction speed of these algorithms, Table 1 shows that the reconstruction speed of PMIMP is slower than that of EMPA, CoSaMP and 
OMP but faster than that of KF-CS, LS-CS, KFLS-CS and Modified-CS for all the frames of the phantom image sequence. From Table 2 and Table 3, we find that EMPA achieves the fastest reconstruction speed for all the frames of brain CT image sequence and brain MR image sequence. Table 2 and Table 3 also show that PMIMP is faster than CoSaMP, KF-CS, LS-CS, KFLS-CS and Modified-CS for brain CT image sequence and brain MR image sequence reconstruction. As analysed in Section 3.4, the computational complexity of PMIMP is influenced significantly by the number of iterations. As described above, we set the estimated support collection of the previous image as the prior information to guide the current image reconstruction, which can reduce the iterations. Also, the multi-variable sampling scheme is used to enhance the guidance of the prior information to improve the reconstruction accuracy. Moreover, the updating mechanism is designed based on the matching strategies of greedy algorithm which performs quite well in reconstruction speed. Although we take measures to accelerate the reconstruction speed, the necessary number of iterations of PMIMP is still larger than that of OMP and EMPA when the measurement rate is the same. The main reason is that the global searching method needs more iterations when the measurement rate is relatively small.

From Table 1, Table 2 and Table 3, we can also see that the PSNR and SSIM of KF-CS, LS-CS, KFLS-CS, Modified-CS and PMIMP decrease with the frames increasing for all the test image sequences. The similarity of KF-CS, LS-CS, KFLS-CS, Modified-CS and PMIMP is that they all set the estimated support collection of the previous image as the prior information to guide the current image reconstruction process and reduce the computational complexity. However, the estimated support collection of the previous image may contain some errors. If there exist some errors in the prior information, it would lead to reconstruction errors in the current image reconstruction and error accumulation in the later images reconstruction. Nevertheless, multi-variable sampling scheme in PMIMP can enhance the guidance of the prior information, which is beneficial to reduce the errors in the prior information. Also, PMIMP aims to search for the global solution and has a higher reconstruction accuracy. So PMIMP can 
improve the error accumulation problem significantly.

The visual performance [47] of image sequences reconstruction based on these algorithms, which are shown in Fig.7 , are also utilized to demonstrate the superiority of the proposed method PMIMP over other state-of-the-art algorithms. The first column represents the original image with the enclosed rectangle and The right three columns represent the enlarged part corresponding to the enclosed rectangle based on those algorithms. From Fig.7, we find that PMIMP provides the reconstruction results with a best visual, especially from the local enlarged parts of the reconstructed images. Although the compared state-ofthe-art algorithms have high PSNR for image sequences reconstruction, they are more likely to make more noisy and blurry and generate artefacts in the areas of edges. In conclusion, all the experimental results show that PMIMP outperforms the state-of-the-art algorithms in reconstruction quality and has a reasonable reconstruction speed.

\section{Conclusion}

This paper proposes a novel predicted multi-variable intelligent matching pursuit algorithm for image sequences reconstruction. The estimated support collection of the previous image is set as the prior information to guide the current image reconstruction, which contributes a lot to reduce the computational complexity and improve the reconstruction accuracy with fewer measurements. Under the guidance of the prior information, PMIMP solves the L0 minimization essentially by utilizing the superior performance of intelligent optimization algorithm in solving combinatorial optimization problems. Also, PMIMP accelerates the reconstruction speed by introducing the matching strategies of greedy algorithm. The multi-variable sampling scheme is also used to enhance the guidance of prior information and improve the reconstruction performance. Experimental results on phantom image sequence, brain CT image sequence and brain MR image sequence demonstrate that PMIMP outperforms the state-ofthe-art algorithms, including OMP, CoSaMP, EMPA, KF-CS, LS-CS, KFLS-CS, 
Table 1: Reconstruction performance of OMP, CoSaMP, EMPA, KF-CS, LSCS, KFLS-CS, Modified-CS and PMIMP for phantom image sequences with the measurement rate fixed as 0.3. The evaluate indexes are PSNR (dB), SSIM and Time (Sec.).

\begin{tabular}{|c|c|c|c|c|c|}
\hline & Algorithm & Fig.4.(a) & Fig.4.(b) & Fig.4.(c) & Fig.4.(d) \\
\hline \multirow{8}{*}{$\operatorname{PSNR}(\mathrm{dB})$} & OMP & 35.3029 & 30.3944 & 29.3495 & 31.6779 \\
\hline & CoSaOMP & 40.7377 & 41.2062 & 48.3543 & 39.6142 \\
\hline & EMPA & 30.1307 & 30.0778 & 29.2935 & 29.6408 \\
\hline & $\mathrm{KF}-\mathrm{CS}$ & 36.8758 & 30.6431 & 24.6974 & 22.9676 \\
\hline & LS-CS & 37.3376 & 30.9702 & 22.3001 & 22.3655 \\
\hline & KFLS-CS & 36.8758 & 33.6719 & 31.4116 & 30.3330 \\
\hline & Modified-CS & 60.9655 & 21.5291 & 18.8761 & 18.6720 \\
\hline & Proposed PMIMP & 345.5257 & 331.2036 & 319.7765 & 307.3299 \\
\hline \multirow{7}{*}{ SSIM } & OMP & 0.9951 & 0.8708 & 0.8617 & 0.8736 \\
\hline & CoSaOMP & 0.9959 & 0.9965 & 0.9925 & 0.9231 \\
\hline & EMPA & 0.8329 & 0.8324 & 0.7615 & 0.7979 \\
\hline & $\mathrm{KF}-\mathrm{CS}$ & 0.9866 & 0.9732 & 0.9122 & 0.8769 \\
\hline & LS-CS & 0.9866 & 0.9843 & 0.9528 & 0.9515 \\
\hline & KFLS-CS & 0.9899 & 0.9826 & 0.9612 & 0.9618 \\
\hline & Modified-CS & 0.9955 & 0.8911 & 0.7555 & 0.6784 \\
\hline \multirow{10}{*}{ Runtime(Sec.) } & Proposed PMIMP & 1.0000 & 1.0000 & 1.0000 & 1.0000 \\
\hline & OMP & 1.3095 & 2.3302 & 1.9945 & 1.8643 \\
\hline & CoSaOMP & 1.7478 & 1.6330 & 2.1626 & 1.0395 \\
\hline & EMPA & 1.7142 & 2.6498 & 1.9596 & 2.0285 \\
\hline & $\mathrm{KF}-\mathrm{CS}$ & 79.8797 & 75.3342 & 79.3065 & 81.2341 \\
\hline & $\mathrm{LS}-\mathrm{CS}$ & 54.2956 & 55.3247 & 54.3982 & 54.0019 \\
\hline & KFLS-CS & 59.8639 & 59.0047 & 58.9215 & 59.0092 \\
\hline & Modified-CS & 1310.1789 & 1321.3917 & 1302.3349 & 1315.4752 \\
\hline & Proposed PMIMP & 5.7345 & 5.9938 & 6.0204 & 5.7763 \\
\hline & Algorithm & Fig.4.(e) & Fig.4.(f) & Fig.4.(g) & Fig.4.(h) \\
\hline \multirow{8}{*}{$\operatorname{PSNR}(\mathrm{dB})$} & OMP & 28.2755 & 29.7997 & 32.0038 & 33.6883 \\
\hline & CoSaOMP & 42.3181 & 40.5928 & 46.0638 & 48.5230 \\
\hline & EMPA & 29.2647 & 26.8250 & 27.4184 & 26.4448 \\
\hline & $\mathrm{KF}-\mathrm{CS}$ & 18.3941 & 17.2486 & 15.8280 & 15.0472 \\
\hline & LS-CS & 19.1480 & 19.0205 & 18.0225 & 17.2368 \\
\hline & KFLS-CS & 24.8242 & 26.6209 & 20.8070 & 20.7701 \\
\hline & Modified-CS & 18.7730 & 19.3015 & 20.6473 & 22.5818 \\
\hline & Proposed PMIMP & 303.3325 & 107.4318 & 89.5215 & 76.3349 \\
\hline \multirow{6}{*}{ SSIM } & OMP & 0.8341 & 0.8779 & 0.8853 & 0.9454 \\
\hline & CoSaOMP & 0.9963 & 0.9978 & 0.9939 & 0.9987 \\
\hline & EMPA & 0.7701 & 0.7456 & 0.7039 & 0.6381 \\
\hline & $\mathrm{KF}-\mathrm{CS}$ & 0.7886 & 0.7020 & 0.7020 & 0.6776 \\
\hline & $\mathrm{LS}-\mathrm{CS}$ & 0.8777 & 0.8426 & 0.8045 & 0.7995 \\
\hline & KFLS-CS & 0.9196 & 0.9288 & 0.8746 & 0.8764 \\
\hline \multirow{10}{*}{ Runtime(Sec.) } & Modified-CS & 0.6396 & 0.6303 & 0.6438 & 0.6642 \\
\hline & Proposed PMIMP & 1.0000 & 1.0000 & 0.9995 & 0.9992 \\
\hline & OMP & 2.2071 & 1.9718 & 2.1239 & 1.7143 \\
\hline & CoSaOMP & 1.6251 & 1.5185 & 1.7098 & 1.2204 \\
\hline & EMPA & 2.0957 & 2.0206 & 2.8607 & 1.4684 \\
\hline & $\mathrm{KF}-\mathrm{CS}$ & 80.9236 & 78.4657 & 79.9124 & 79.2268 \\
\hline & LS-CS & 55.3645 & 53.0718 & 53.7759 & 54.0196 \\
\hline & KFLS-CS & 58.9114 & 57.3019 & 58.0443 & 59.2984 \\
\hline & Modified-CS & 1299.0346 & 1302.4407 & 1310.2246 & 1313.3325 \\
\hline & Proposed PMIMP & 6.2325 & 6.9937 & 10.8564 & 11.7358 \\
\hline
\end{tabular}


Table 2: Reconstruction performance of OMP, CoSaMP, EMPA, KF-CS, LSCS, KFLS-CS, Modified-CS and PMIMP for brain CT image sequences with the measurement rate fixed as 0.4. The evaluate indexes are PSNR (dB), SSIM and Time (Sec.).

\begin{tabular}{|c|c|c|c|c|c|}
\hline & Algorithm & Fig.5.(a) & Fig.5.(b) & Fig.5.(c) & Fig.5.(d) \\
\hline \multirow{8}{*}{$\operatorname{PSNR}(\mathrm{dB})$} & OMP & 32.2677 & 30.1474 & 28.9431 & 27.0468 \\
\hline & CoSaOMP & 29.3565 & 29.0777 & 26.0188 & 27.3607 \\
\hline & EMPA & 36.5050 & 36.0334 & 34.6147 & 33.1259 \\
\hline & KF-CS & 47.1368 & 45.4938 & 43.6425 & 42.6077 \\
\hline & LS-CS & 47.1357 & 45.3359 & 43.4611 & 42.2257 \\
\hline & KFLS-CS & 47.1368 & 45.3791 & 43.7155 & 42.6640 \\
\hline & Modified-CS & 51.1024 & 23.1731 & 21.6475 & 20.8115 \\
\hline & Proposed PMIMP & 313.7525 & 311.6032 & 309.7215 & 65.8082 \\
\hline \multirow{7}{*}{ SSIM } & OMP & 0.6551 & 0.5833 & 0.5408 & 0.4744 \\
\hline & CoSaOMP & 0.6834 & 0.6752 & 0.6362 & 0.6244 \\
\hline & EMPA & 0.9152 & 0.8904 & 0.8671 & 0.8741 \\
\hline & $\mathrm{KF}-\mathrm{CS}$ & 0.9448 & 0.8963 & 0.8317 & 0.7734 \\
\hline & LS-CS & 0.9448 & 0.8984 & 0.8479 & 0.7792 \\
\hline & KFLS-CS & 0.9448 & 0.9004 & 0.8583 & 0.8024 \\
\hline & Modified-CS & 0.9935 & 0.8289 & 0.7779 & 0.7490 \\
\hline \multirow{10}{*}{ Runtime(Sec.) } & Proposed PMIMP & 1.0000 & 1.0000 & 1.0000 & 0.9990 \\
\hline & $\mathrm{OMP}$ & 6.2389 & 6.7083 & 5.5441 & 5.6250 \\
\hline & CoSaOMP & 46.2100 & 45.2274 & 43.5861 & 47.6396 \\
\hline & EMPA & 2.1111 & 1.9959 & 2.0552 & 2.0841 \\
\hline & $\mathrm{KF}-\mathrm{CS}$ & 133.1188 & 131.5463 & 129.0492 & 135.7246 \\
\hline & LS-CS & 133.4220 & 132.0022 & 129.8947 & 134.2673 \\
\hline & KFLS-CS & 126.2407 & 125.7602 & 125.3918 & 128.0031 \\
\hline & Modified-CS & 1249.8750 & 1233.6502 & 1245.3021 & 1254.7682 \\
\hline & Proposed PMIMP & 48.9306 & 45.3350 & 47.3041 & 49.8965 \\
\hline & Algorithm & Fig.5.(e) & Fig.5.(f) & Fig.5.(g) & Fig.5.(h) \\
\hline \multirow{9}{*}{$\operatorname{PSNR}(\mathrm{dB})$} & OMP & 26.7885 & 25.3736 & 24.8625 & 24.7011 \\
\hline & CoSaOMP & 28.2573 & 26.7851 & 24.3437 & 26.1516 \\
\hline & EMPA & 32.7888 & 34.2600 & 33.1097 & 32.7257 \\
\hline & $\mathrm{KF}-\mathrm{CS}$ & 41.2372 & 40.3894 & 39.2636 & 36.3138 \\
\hline & LS-CS & 41.1453 & 40.1226 & 38.4893 & 35.3262 \\
\hline & KFLS-CS & 41.6516 & 40.6993 & 39.3142 & 35.9848 \\
\hline & Modified-CS & 20.3595 & 19.7429 & 18.9292 & 19.4461 \\
\hline & Proposed PMIMP & 52.4132 & 44.4149 & 44.2461 & 41.4460 \\
\hline & OMP & 0.4828 & 0.4505 & 0.4515 & 0.4565 \\
\hline \multirow{5}{*}{ SSIM } & CoSaOMP & 0.6279 & 0.5969 & 0.5799 & 0.5860 \\
\hline & EMPA & 0.8661 & 0.8815 & 0.8943 & 0.9006 \\
\hline & $\mathrm{KF}-\mathrm{CS}$ & 0.6937 & 0.6461 & 0.5663 & 0.5057 \\
\hline & LS-CS & 0.7265 & 0.6577 & 0.5947 & 0.5011 \\
\hline & KFLS-CS & 0.7555 & 0.7061 & 0.6262 & 0.5448 \\
\hline \multirow{10}{*}{ Runtime(Sec.) } & Modified-CS & 0.7294 & 0.7034 & 0.6770 & 0.6681 \\
\hline & Proposed PMIMP & 0.9931 & 0.9615 & 0.9565 & 0.9399 \\
\hline & OMP & 5.6205 & 5.6855 & 5.7457 & 5.5964 \\
\hline & CoSaOMP & 48.1433 & 40.1073 & 38.1125 & 39.1752 \\
\hline & EMPA & 2.0738 & 2.2222 & 2.1868 & 1.3950 \\
\hline & $\mathrm{KF}-\mathrm{CS}$ & 133.7243 & 131.6592 & 134.3342 & 133.2984 \\
\hline & LS-CS & 133.2574 & 132.4423 & 135.1103 & 133.8437 \\
\hline & KFLS-CS & 126.4125 & 125.3384 & 126.8569 & 127.0128 \\
\hline & Modified-CS & 1231.3428 & 1241.5791 & 1246.7708 & 1249.0078 \\
\hline & Proposed PMIMP & 43.3325 & 45.6052 & 43.2238 & 47.8495 \\
\hline
\end{tabular}


Table 3: Reconstruction performance of OMP, CoSaMP, EMPA, KF-CS, LSCS, KFLS-CS, Modified-CS and PMIMP for brain MR image sequences with the measurement rate fixed as 0.4. The evaluate indexes are PSNR (dB), SSIM and Time (Sec.).

\begin{tabular}{|c|c|c|c|c|c|}
\hline & Algorithm & Fig.6.(a) & Fig.6.(b) & Fig.6.(c) & Fig.6.(d) \\
\hline \multirow{8}{*}{$\operatorname{PSNR}(\mathrm{dB})$} & OMP & 27.3853 & 28.3399 & 28.3087 & 28.5572 \\
\hline & CoSaOMP & 30.1940 & 30.9975 & 31.1980 & 31.1142 \\
\hline & EMPA & 28.7096 & 31.1382 & 29.6749 & 31.7233 \\
\hline & $\mathrm{KF}-\mathrm{CS}$ & 44.1818 & 43.6616 & 43.3851 & 43.0963 \\
\hline & LS-CS & 43.1143 & 43.5607 & 43.4191 & 43.3237 \\
\hline & KFLS-CS & 43.1153 & 43.4674 & 43.3796 & 43.5755 \\
\hline & Modified-CS & 46.2245 & 26.2259 & 24.2790 & 24.2120 \\
\hline & Proposed PMIMP & 259.4751 & 267.8522 & 219.9325 & 214.8422 \\
\hline \multirow{7}{*}{ SSIM } & OMP & 0.8092 & 0.8307 & 0.8150 & 0.8197 \\
\hline & CoSaOMP & 0.8745 & 0.8785 & 0.8814 & 0.8786 \\
\hline & EMPA & 0.9052 & 0.9102 & 0.9042 & 0.9094 \\
\hline & $\mathrm{KF}-\mathrm{CS}$ & 0.9274 & 0.9147 & 0.8921 & 0.8637 \\
\hline & LS-CS & 0.9284 & 0.9255 & 0.9104 & 0.8986 \\
\hline & KFLS-CS & 0.9284 & 0.9136 & 0.9020 & 0.9261 \\
\hline & Modified-CS & 0.9952 & 0.8821 & 0.8485 & 0.8390 \\
\hline \multirow{10}{*}{ Runtime(Sec.) } & Proposed PMIMP & 1.0000 & 1.0000 & 1.0000 & 1.0000 \\
\hline & OMP & 30.0831 & 30.0994 & 32.9009 & 30.9347 \\
\hline & CoSaOMP & 179.3817 & 181.4019 & 143.2110 & 151.2489 \\
\hline & EMPA & 6.8130 & 4.9547 & 5.5423 & 6.7143 \\
\hline & $\mathrm{KF}-\mathrm{CS}$ & 796.1727 & 785.2950 & 789.4307 & 791.3340 \\
\hline & $\mathrm{LS}-\mathrm{CS}$ & 892.7455 & 873.2085 & 884.3927 & 891.3042 \\
\hline & KFLS-CS & 633.6324 & 625.9341 & 631.2401 & 642.3306 \\
\hline & Modified-CS & 4683.8234 & 4659.0458 & 4599.4602 & 4615.1096 \\
\hline & Proposed PMIMP & 96.7083 & 89.3024 & 95.3342 & 93.2048 \\
\hline & Algorithm & Fig.6.(e) & Fig.6.(f) & Fig.6.(g) & Fig.6.(h) \\
\hline \multirow{9}{*}{$\operatorname{PSNR}(\mathrm{dB})$} & OMP & 28.3976 & 28.4620 & 28.1593 & 27.7871 \\
\hline & CoSaOMP & 25.9564 & 31.5146 & 28.0292 & 31.3147 \\
\hline & EMPA & 31.9246 & 31.7087 & 31.5786 & 31.2722 \\
\hline & $\mathrm{KF}-\mathrm{CS}$ & 42.5287 & 42.0795 & 41.2980 & 40.7564 \\
\hline & LS-CS & 42.9142 & 42.4548 & 41.7029 & 41.0450 \\
\hline & KFLS-CS & 42.9132 & 42.6823 & 41.8923 & 41.4761 \\
\hline & Modified-CS & 23.4194 & 22.7432 & 22.4175 & 22.6714 \\
\hline & Proposed PMIMP & 78.5178 & 62.1443 & 52.8877 & 43.6174 \\
\hline & OMP & 0.8134 & 0.8310 & 0.8310 & 0.8310 \\
\hline \multirow{5}{*}{ SSIM } & CoSaOMP & 0.8887 & 0.8959 & 0.8932 & 0.8948 \\
\hline & EMPA & 0.9168 & 0.9191 & 0.9237 & 0.9217 \\
\hline & $\mathrm{KF}-\mathrm{CS}$ & 0.8316 & 0.7965 & 0.7466 & 0.7111 \\
\hline & $\mathrm{LS}-\mathrm{CS}$ & 0.8826 & 0.8482 & 0.8090 & 0.7658 \\
\hline & KFLS-CS & 0.8820 & 0.8603 & 0.8199 & 0.7944 \\
\hline \multirow{10}{*}{ Runtime(Sec.) } & Modified-CS & 0.8254 & 0.8141 & 0.7960 & 0.7995 \\
\hline & Proposed PMIMP & 0.9999 & 0.9997 & 0.9992 & 0.9932 \\
\hline & OMP & 32.5147 & 31.7172 & 38.7773 & 32.0074 \\
\hline & CoSaOMP & 176.3626 & 158.2339 & 191.9748 & 144.6778 \\
\hline & EMPA & 6.9919 & 6.3365 & 6.5292 & 5.9541 \\
\hline & $\mathrm{KF}-\mathrm{CS}$ & 795.6328 & 791.3407 & 801.3329 & 789.0039 \\
\hline & LS-CS & 879.6237 & 895.5569 & 883.0938 & 891.4075 \\
\hline & KFLS-CS & 633.0172 & 629.5895 & 635.3019 & 632.4495 \\
\hline & Modified-CS & 4654.0927 & 4597.2096 & 4671.9035 & 4691.3047 \\
\hline & Proposed RMIMP & 101.4405 & 91.4095 & 93.2043 & 95.9783 \\
\hline
\end{tabular}




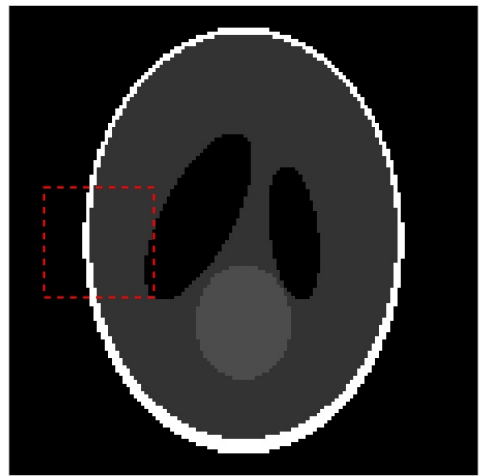

Original image

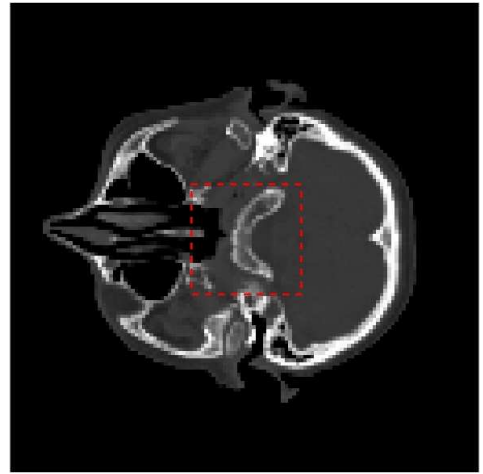

Original image

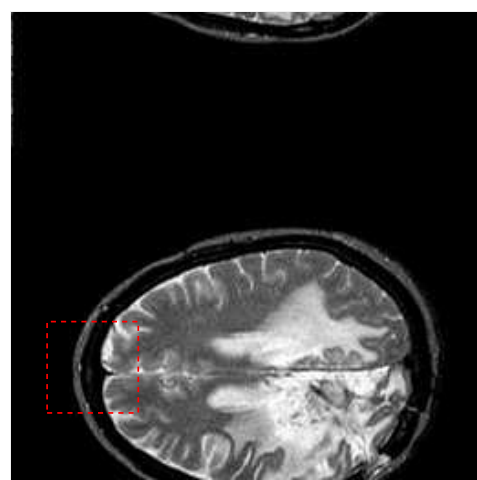

Original image

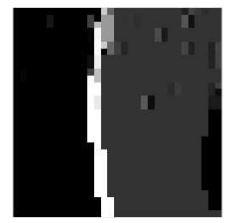

OMP

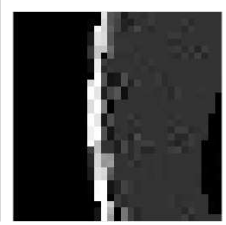

$\mathrm{KF}-\mathrm{CS}$

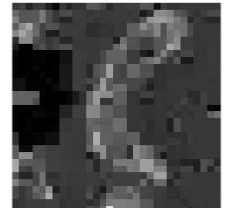

OMP

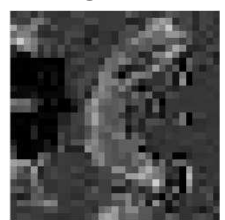

$\mathrm{KF}-\mathrm{CS}$

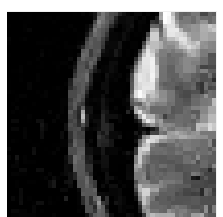

OMP

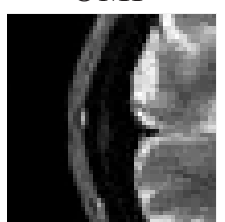

$\mathrm{KF}-\mathrm{CS}$

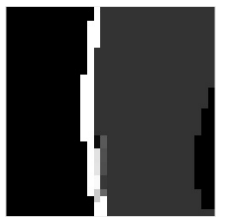

CoSaMP

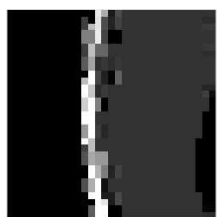

LS-CS

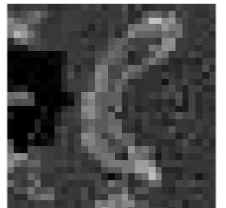

CoSaMP

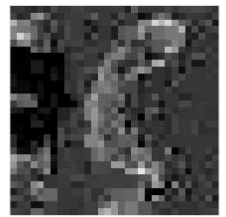

LS-CS

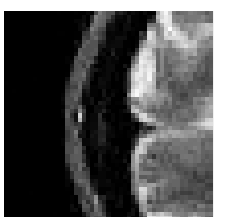

CoSaMP

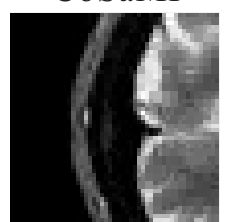

LS-CS

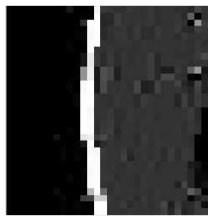

EMPA

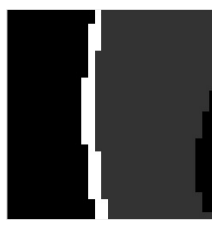

PMIMP

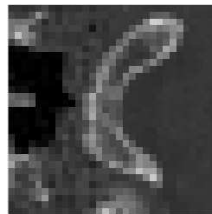

EMPA

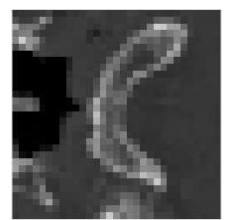

PMIMP

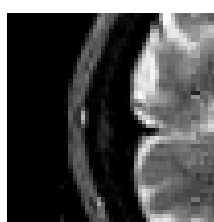

EMPA

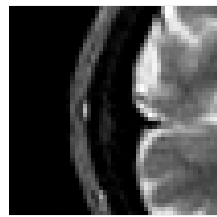

PMIMP

Figure 7: Visual performance of OMP, CoSaMP, EMPA, KF-CS, LS-CS and PMIMP for phantom image sequences, brain $\mathrm{CT}$ image sequences and brain MR image sequences. The first column shows the original image with the enclosed rectangle. The right three columns show the enlarged part corresponding to the enclosed rectangle based on those algorithms. 
Modified-CS, both on PSNR, SSIM and visual quality. One possible extension of our work would be to use multi-level wavelet decomposition to reduce the measurement rate and introduce more effective update mechanism into PMIMP to further reduce the computational complexity.

\section{ACKNOWLEDGMENT}

This work is financially supported by National Science Foundations of China (No.61174016) and (No.61171197).

\section{References}

[1] D. L. Donoho, "compressed sensing", IEEE Transactions on Information Theory, vol. 52, no.4, (Apr. 2006) pp. 1289-1306.

[2] E. Candes, M. Wakin, "an introduction to compressive sampling [a sensing/sampling paradigm that goes against the common knowledge in data acquisition]", IEEE Signal Process Mag, vol. 25, (Apr. 2008) pp. 21-30.

[3] R. G. Baraniuk, V. Cevher, M. F. Duarte, C. Hegde, "model-based compressive sensing", IEEE Transactions on Information Theory, vol. 56, no.4, (Apr. 2010) pp. 1982-2001.

[4] M. Wakin, J. Laska, M. Duarte, D. Baron, S. Sarvotham, D. Takhar, K. Kelly, R. G. Baraniuk, "compressive imaging for video representation and coding", in: Picture Coding Symposium,, Vol. 1, Apr. 2006.

[5] U. Gamper, P. Boesiger, S. Kozerke, "compressed sensing in dynamic mri", Magnetic Resonance in Medicine, vol. 59, no. 2, (Feb. 2008) pp.365-373.

[6] H. Jung, K. Sung, K. S. Nayak, E. Y. Kim, J. C. Ye, "k-t focuss: A general compressed sensing framework for high resolution dynamic mri", Magnetic Resonance in Medicine, vol. 61, no. 1, (Jan. 2009) pp. 103-116. 
[7] E. Liu, V. N. Temlyakov, "the orthogonal super greedy algorithm and applications in compressed sensing", IEEE Transactions on Information Theory, vol. 58, no. 4, (Apr. 2012) pp. 2040-2047.

[8] J. Tropp, A. C. Gilbert, et al., "signal recovery from random measurements via orthogonal matching pursuit", IEEE Transactions on Information Theory, vol. 53, no. 12, (Dec. 2007) pp. 4655-4666.

[9] W. Dai, O. Milenkovic, "subspace pursuit for compressive sensing signal reconstruction", IEEE Transactions on Information Theory, vol. 55, no. 5, (May. 2009) pp. 2230-2249.

[10] D. L. Donoho, Y. Tsaig, I. Drori, J.-L. Starck, "sparse solution of underdetermined systems of linear equations by stagewise orthogonal matching pursuit", IEEE Transactions on Information Theory, vol. 58, no.2, (Feb. 2012) pp. 1094-1121.

[11] M. D. Plumbley, M. Bevilacqua, "sparse reconstruction for compressed sensing using stagewise polytope faces pursuit”, in: 2009 16th IEEE International Conference on Digital Signal Processing, (Jul. 2009), pp. 1-8.

[12] H. Huang, A. Makur, "backtracking-based matching pursuit method for sparse signal reconstruction", IEEE Signal Processing Letters, vol. 18, no.7, (Jul. 2011) pp. 391-394.

[13] T. T. Do, L. Gan, N. Nguyen, T. D. Tran, "sparsity adaptive matching pursuit algorithm for practical compressed sensing", in: 2008 42nd IEEE Asilomar Conference on Signals, Systems and Computers,, (Oct. 2008), pp. $581-587$.

[14] D. Needell, J. A. Tropp, " cosamp: Iterative signal recovery from incomplete and inaccurate samples", Applied and Computational Harmonic Analysis, vol. 26, no. 3, (May. 2009) pp. 301-321.

[15] Q. Bai, "analysis of particle swarm optimization algorithm", Computer and information science, vol. 3, no. 1, (2010) pp. 180. 
[16] T. Chen, "a simulative bionic intelligent optimization algorithm: Artificial searching swarm algorithm and its performance analysis", in: 2009. CSO 2009. IEEE International Joint Conference on Computational Sciences and Optimization,, Vol. 2, (Apr. 2009), pp. 864-866.

[17] D. Karaboga, B. Basturk, "artificial bee colony (abc) optimization algorithm for solving constrained optimization problems", in: Foundations of Fuzzy Logic and Soft Computing, 2007, pp. 789-798.

[18] X. Fengmin, W. Shanhe, "a hybrid simulated annealing thresholding algorithm for compressed sensing", Signal Processing, vol. 93, no. 6, (Jun. 2013) pp. 1577-1585.

[19] X. Du, L. Cheng, D. Chen, "a simulated annealing algorithm for sparse recovery by 10 minimization", Neurocomputing, vol. 131, (May. 2014) pp. 98-104.

[20] X. Du, L. Cheng, G. Cheng, "a heuristic search algorithm for the multiple measurement vectors problem", Signal Processing, vol. 100, (Jul. 2014) pp. $1-8$.

[21] D. Li, M. Li, Y. Shen, Y. Wang, Q. Wang, "ga-bfo based signal reconstruction for compressive sensing", in: 2013 IEEE International Conference on Information and Automation (ICIA), (Aug. 2013), pp. 1023-1028.

[22] D. Li, C. Shi, Q. Wang, Y. Shen, Y. Wang, "artificial immune algorithm based signal reconstruction for compressive sensing", in: 2014 IEEE International Instrumentation and Measurement Technology Conference (I2MTC) Proceedings, (May. 2014), pp. 76-81.

[23] M. Sajjad, I. Mehmood, S. W. Baik, "image super-resolution using sparse coding over redundant dictionary based on effective image representations", Journal of Visual Communication and Image Representation vol. 26, (Jan. 2015) pp. 50-65. 
[24] A. B. H. Ali Mosleh, Nizar Bouguila, "image and video spatial superresolution via bandlet-based sparsity regularization and structure tensor", Signal Processing: Image Communication vol. 30, (Jan. 2015) pp. 137-146.

[25] I. Mehmood, M. Sajjad, W. Ejaz, S. W. Baik, "saliency-directed prioritization of visual data in wireless surveillance networks", Information Fusion vol. 24, (Jan. 2015) pp. 16-30.

[26] N. Vaswani, "kalman filtered compressed sensing", in: 15th IEEE International Conference on Image Processing, 2008. ICIP 2008, (Oct. 2008), pp. 893-896.

[27] N. Vaswani, "ls-cs-residual (ls-cs): compressive sensing on least squares residual", IEEE Transactions on Signal Processing, vol. 58, no. 8, (Aug. 2010) pp. 4108-4120.

[28] N. Vaswani, W. Lu, "modified-cs: Modifying compressive sensing for problems with partially known support", IEEE Transactions on Signal Processing, vol. 58, no. 9, (Sep. 2010) pp. 4595-4607.

[29] W. Lu, N. Vaswani, "modified compressive sensing for real-time dynamic mr imaging", in: 2009 16th IEEE International Conference on Image Processing (ICIP), (Nov. 2009), pp. 3045-3048.

[30] W. Lu, T. Li, I. C. Atkinson, N. Vaswani, "modified-cs-residual for recursive reconstruction of highly undersampled functional mri sequences", in: 2011 18th IEEE International Conference on Image Processing (ICIP), (Sep. 2011), pp. 2689-2692.

[31] W. Lu, N. Vaswani, "exact reconstruction conditions for regularized modified basis pursuit", IEEE Transactions on Signal Processing, vol. 60, no. 5, (May. 2012) pp. 2634-2640.

[32] W. Lu, N. Vaswani, "regularized modified bpdn for noisy sparse reconstruction with partial erroneous support and signal value knowledge", IEEE Transactions on Signal Processing, vol. 60, no. 1, (Jan. 2012) pp. 182-196. 
[33] V. Cevher, A. Sankaranarayanan, M. F. Duarte, D. Reddy, R. G. Baraniuk, R. Chellappa, "compressive sensing for background subtraction", in: Computer Vision-ECCV 2008, (Oct. 2008), pp. 155-168.

[34] D. Zonoobi, A. A. Kassim, "on the reconstruction of sequences of sparse signals-the weighted-cs", Journal of Visual Communication and Image Representation, vol. 24, no. 2, (Feb. 2013) pp. 196-202.

[35] J. Wu, F. Liu, L. Jiao, X. Wang, "multivariate pursuit image reconstruction using prior information beyond sparsity", Signal Processing, vol. 93, no. 6, (Jun. 2013) pp. 1662-1672.

[36] D. P. Wipf, B. D. Rao, "sparse bayesian learning for basis selection", IEEE Transactions on Signal Processing, vol. 52, no. 8, (Aug. 2004) pp. 21532164 .

[37] S. S. Chen, D. L. Donoho, M. A. Saunders, "atomic decomposition by basis pursuit", SIAM journal on scientific computing, vol. 20, no. 1, (Jul. 1998) pp. $33-61$.

[38] C. Yan, Y. Zhang, J. Xu, F. Dai, J. Zhang, Q. Dai, F. Wu, "efficient parallel framework for hevc motion estimation on many-core processors", IEEE Transactions on Circuits and Systems for Video Technology, vol. 24, no. 12, (Dec. 2014) pp. 2077-2089.

[39] C. Yan, Y. Zhang, J. Xu, F. Dai, L. Li, Q. Dai, F. Wu, "a highly parallel framework for hevc coding unit partitioning tree decision on many-core processors", IEEE Signal Processing Letters, vol. 21, no. 5, (May. 2014) pp. $573-576$.

[40] C. Yan, Y. Zhang, F. Dai, J. Zhang, L. Li, Q. Dai, "efficient parallel hevc intra-prediction on many-core processor", Electronics Letters vol. 50, no. 11, (May. 2014) pp. 805-806.

[41] J. Wu, F. Liu, L. Jiao, X. Wang, B. Hou, "multivariate compressive sensing for image reconstruction in the wavelet domain: using scale mixture mod- 
els", IEEE Transactions on Image Processing, vol. 20, no. 12, (Dec. 2011) pp. 3483-3494.

[42] H. Mühlenbein, M. Schomisch, J. Born, "the parallel genetic algorithm as function optimizer", Parallel computing, vol. 17, no. 6, (Sep. 1991) pp. 619-632.

[43] M. Lundy, A. Mees, "convergence of an annealing algorithm", Mathematical programming, vol. 34, no. 1, (1) (Jan. 1986) pp. 111-124.

[44] L. Gan, "block compressed sensing of natural images", in: 2007 15th IEEE International Conference on Digital Signal Processing,, Jul. 2007, pp. 403406.

[45] D. Needell, "topics in compressed sensing", arXiv preprint arXiv:0905.4482.

[46] Z. Wang, A. C. Bovik, H. R. Sheikh, E. P. Simoncelli, "image quality assessment: from error visibility to structural similarity", IEEE Transactions on Image Processing, vol. 13, no. 4, (Apr. 2004) pp. 600-612.

[47] M. Sajjad, I. Mehmood, N. Abbas, S. W. Baik, "basis pursuit denoisingbased image superresolution using a redundant set of atoms", Signal, Image and Video Processing vol. 10, no. 1, (Jan. 2016) pp. 181-188. 